\title{
High-resolution fracture recognition from seismic data - applying oil and gas technology to mining
}

\author{
R.H.J. Oppermann OPPtimal Exploration and Development Pty Ltd, Australia
}

\begin{abstract}
During the last two decades, volume interpretation techniques have revolutionised traditional 3D seismic interpretation workflows in the oil and gas industry. While traditional interpretation mainly relies on an interpreter to work through a large volume of data to identify and manually characterise subsurface geology and resources, volume interpretation allows to filter and classify subsurface features and extract 3D geological information through automated processes at a much faster pace, with higher accuracy and at higher resolution.
\end{abstract}

This paper presents innovative techniques and workflows in structural volume interpretation that have been developed and proven extremely successful in oil and gas projects. More recently, this technology has been applied to mining projects. The workflows integrate highest-resolution, automated fracture network mapping results from 3D seismic data with the detailed calibration and review of various drilling, mining and production data, allowing to delineate fluid barriers, fluid conduits, fault-related mineralisations or drilling and mining hazards in the subsurface. From this, groundbreaking insights can be gained into the $3 D$ distribution of fracture networks, and how these can affect drilling and mining activities and the production of resources.

Examples from oil and gas and mining projects around the world demonstrate that these new techniques can provide a step-change in understanding drilling, production and safety issues in existing wells or mines. Furthermore, they can be utilised to optimise future resource activities and recoveries, and increase the safety of future operations.

Automated fracture extraction techniques challenge widespread perceptions of what is seismically resolvable from 3D seismic data. Fault and fracture networks can not only be identified faster and more reliably, but also at a much higher resolution than achieved by other current seismic methods. With the increased resolution, much greater fault/fracture densities are identified than previously mapped or recognised, and a better understanding of structural geometries and fault populations can be gained.

A focused application of the new technology workflows can deliver increased recoveries from resources. It can also result in safer, cheaper and more successful drilling and mining operations. As such, these new techniques are proposed as Best Practice tools for both mining and oil and gas resource development planning and execution.

\section{$1 \quad$ Resources and fractures}

Fault and fracture networks can have significant effects on drilling, mining and the safety of resource operations, and can also significantly impact resource recovery and productivity.

Fracture penetrations can cause drilling or mining problems, potentially resulting in Non-Productive Time or safety incidents. Fracture intersections can lead to geomechanical issues in boreholes (wellbore instability, breakouts, casing damage due to slippage along reactivated fault planes, etc.) or mines (roof instability/ collapse, rockburst, fault reactivation during tunnelling, etc.). Fracture penetrations can also lead to incidents related to lost circulation or gas kicks in wells, water inflow or outbursts in mines and even total well, rig or mine losses, associated with possible loss of lives. For example, all fatal outbursts in Australian collieries have occurred on faults. Fractures can also directly control the emplacement of both mining and 
oil and gas resources, by providing pathways for fluids to migrate from source areas to traps (sourcetransport-trap mechanism). Many ore deposits are directly controlled by fractures, in particular hydrothermal ore deposits, most of which precipitate from hydrothermal fluids that move through fractured rocks.

Fractures often directly affect resource production outcomes. In oil and gas reservoirs, fracture networks can act as either fluid barriers or fluid conduits in a reservoir. In conventional, matrix-producing reservoirs, faults can offset productive layers and represent zones of reduced porosity and permeability that can compartmentalise the reservoir and provide baffles or barriers to fluid flow in the reservoir (Antonellini and Aydin, 1994). In fractured reservoirs, however, where most of the permeability is caused by fracturing, faults can provide efficient conduits for fluid flow (Maerten et al., 2006).

In coal mining, faults can cause major interruptions to production and can even affect the economic viability of a coal mine (Cocker et al., 1997). By offsetting coal seams, faults can cause costly machinery readjustments during longwall or room-and-pillar mining. Drill-hole data and high-resolution 3D seismic data are often acquired at the early stages of development. One of the key purposes for acquiring the data is to identify faults and to be able to avoid the most faulted areas during mining. Ground control strategies typically include mine designs that minimise fault exposure (Molinda and Ingram, 1990). Interpreted faults are carefully integrated with well data and considered during mine design and detailed mine planning, as well as during coal extraction (Peters and Hearn, 2001). Faults encountered in the subsurface during mining are evaluated in detail and compared with faults mapped from 3D seismic to calibrate and improve fault prediction capabilities of the (manual) seismic interpretation effort. The early detection of faults on 3D seismic data also allows coal mining companies to degasify the coal seam in advance of mining operations (Gochioco and Cotten, 1989). Detailed fault mapping and subsequent pre-drainage of mine gas is an important commercial and also safety-increasing activity (Cocker et al., 1997).

In ore mining, detailed fracture network description and property quantification (e.g. distribution, orientation, size and intensity of fractures) are of key importance for fractured rock mass characterisation and mineral resource evaluations.

In most resource industries, it is critical to improve the understanding, detection, modelling and prediction of fault and fracture networks. Nearly all natural resources are affected by fractures, yet the effects of fractures are often poorly understood and, in the oil and gas industry, are often also underestimated or even denied ('fracture denial'; Nelson, 2001). The key problem for the development of fractured resources is the difficulty to visualise the exact location and geometry of fractures, especially as a large component of the fracture network is typically beneath the imaging resolution of most geophysical methods (Lonergan et al., 2007).

Novel techniques and workflows in Automated Fracture Extraction (AFE) from 3D seismic data, however, offer new and exciting opportunities to visualise fracture networks at extremely high resolution (Figure 1). The workflows provide a completely new basis for the reliable identification and quantifiable prediction of fracture networks in the subsurface.

\section{$2 \quad$ Automated fracture extraction}

3D seismic data is the key tool in the oil and gas industry to map and predict reservoir properties and fault and fracture networks away from well control. The interpretation of 3D seismic data and the (often sparse) well data serve as key controls for the construction of detailed reservoir models.

Given the important role that fault and fracture networks play in hydrocarbon reservoirs, various seismic processing techniques and software packages focused on 3D fault visualisation, auto-extraction and also semi-automated fault picking have been developed in recent years. These automated fault detection techniques aim to support or replace manual fault mapping efforts, which are not only labour-intensive and time-consuming (Admasu et al., 2006), but also subjective. 

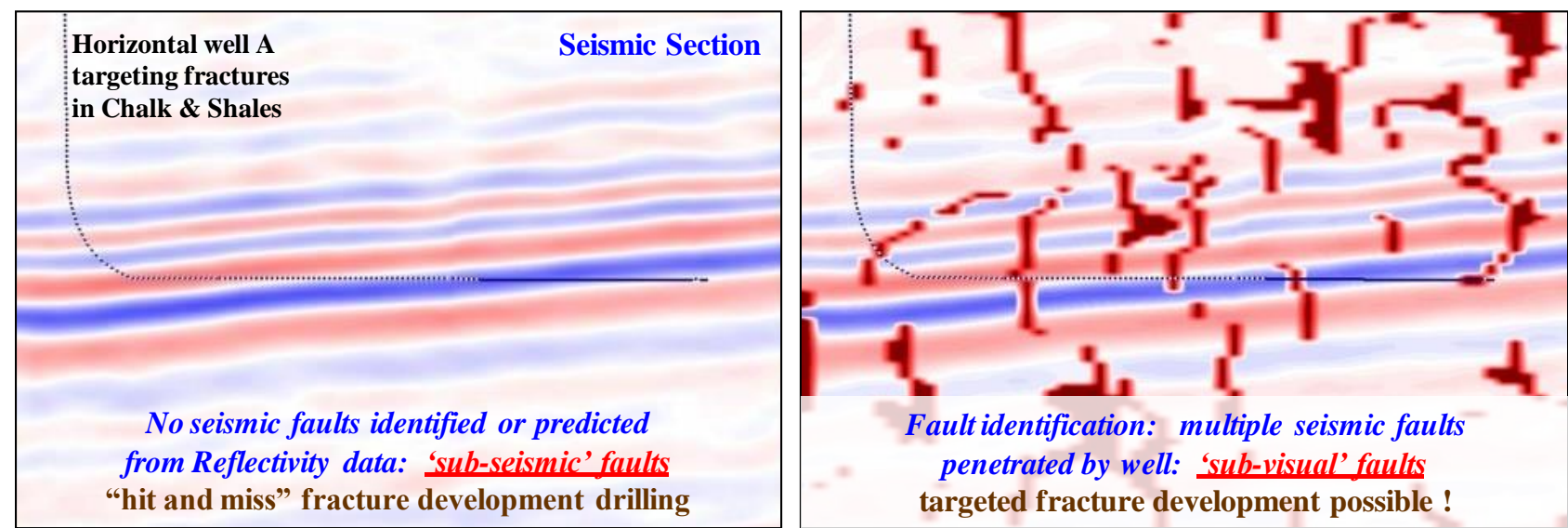

Figure 1 High-resolution fault extraction visualises small-scale spatial changes in amplitude, frequency or phase content of 3D seismic data, and challenges perceptions of what can and cannot be identified with seismic data. Comparison and calibration of seismic fault extractions with faults identified in wells (from e.g. core, image logs/diameter or log correlation) helps to ground-truth extractions and assess the true seismic fault resolution of a particular data set at objective level

Various attributes are in use for imaging discontinuities in seismic data, e.g. coherence, semblance, curvature, similarity, dip and azimuth, frequency variability, seismic texture, etc. These attributes typically identify and enhance spatial discontinuities that are computed at every data point within a seismic data cube. For a description of attributes and a detailed account of the advances made in the automation of seismic fault interpretation, reference is made to the publication by Pepper and Bejarano (2005).

While the identified seismic discontinuities most times represent fault surfaces, they can also be related to other geologic features (channel edges, dykes, etc.) or noise (acquisition/processing artefacts). Noisecontamination of seismic data can be addressed by running spatial filters that remove the noise but retain the geometric detail such as small-scale faults breaks (Chopra and Marfurt, 2007). Noise reduction can for example be achieved without degradation to the fault expression by data conditioning with structureoriented smoothing utilising edge preservation (Hoecker and Fehmers, 2002).

Most discontinuity processing workflows follow a similar approach, i.e. potential volume conditioning with noise cancellation, followed by automatic discontinuity delineation, conversion into 3D objects and calibration and analysis of these objects.

\section{1 Calibration}

It is of key importance to confirm that discontinuity extractions represent structural features, rather than artefacts. A number of calibration steps help with this validation process (Oppermann, 2010):

- Visual inspection of extraction results on sections, time slices and in volume view (Figure 2) helps to determine if obvious faults/horizon offsets are successfully identified, if fault patterns and geometries are meaningful and if features are consistently being identified, also when comparing different algorithm results. Visual inspection will also help to assess whether the data is affected by noise pollution and if structure-oriented filtering of the data is required.

- Calibration against other geophysical data, e.g. structural highlighting data (e.g. Dip, Azi, DipAzi, etc., Figure 3), often reveals a good match between seismic discontinuities and features indicated by other geophysical data. Fracture extraction, however, usually delivers a much higher resolution than achieved by other methods.

- Calibration against previous (manual) fault interpretation typically shows a good match between extracted seismic discontinuities and faults mapped by an interpreter. High-resolution extraction, 
however, identifies a lot more (smaller-scale) discontinuities than have been visually mapped by an interpreter, due to the higher resolution achieved by auto-extraction (Figure 3).

- Calibration against faults and fractures identified from well data, e.g. log correlation, core, image logs, dipmeter, etc. typically plays a key role in proving that seismic discontinuities are geological features, as these well data-based interpretations are totally independent of 3D seismic data.
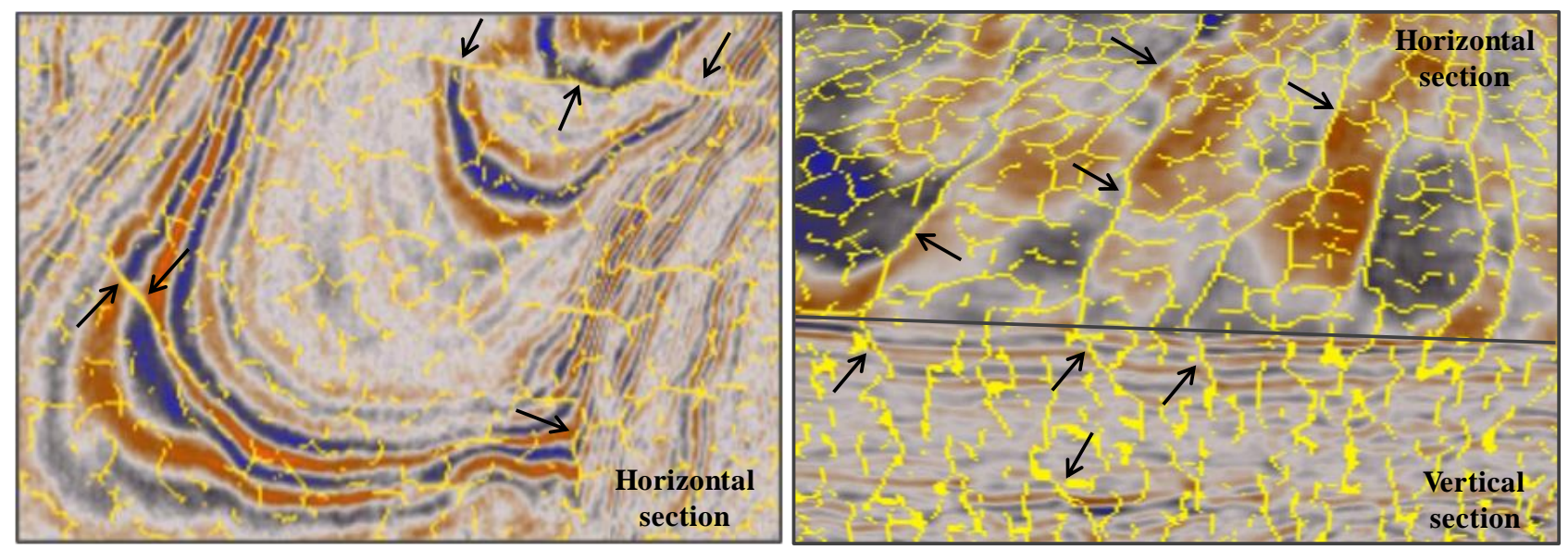

Figure 2 Visual inspection of fault extraction results confirms that faults with larger offsets (arrows) have been successfully delineated. Also, smaller scale faults without obvious offsets are identified, most of which an Interpreter could not pick confidently and reliably by visual means only 


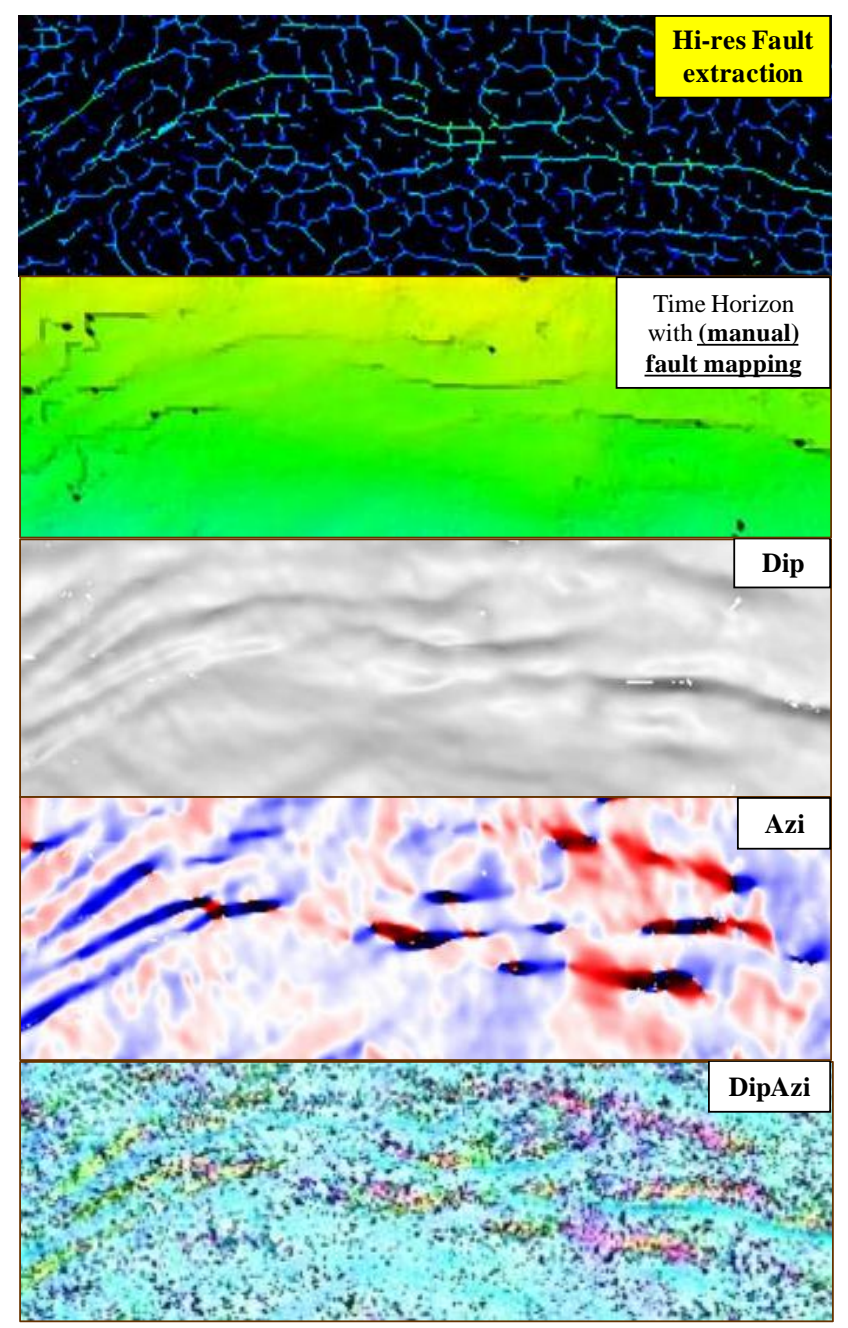

Figure 3 Comparison of high resolution fault extraction results with manual fault mapping results, and other structural highlighting data. Fault extraction delivers a much higher resolution than, e.g. visual fault mapping, Dip, Azi or DipAzi volumes 


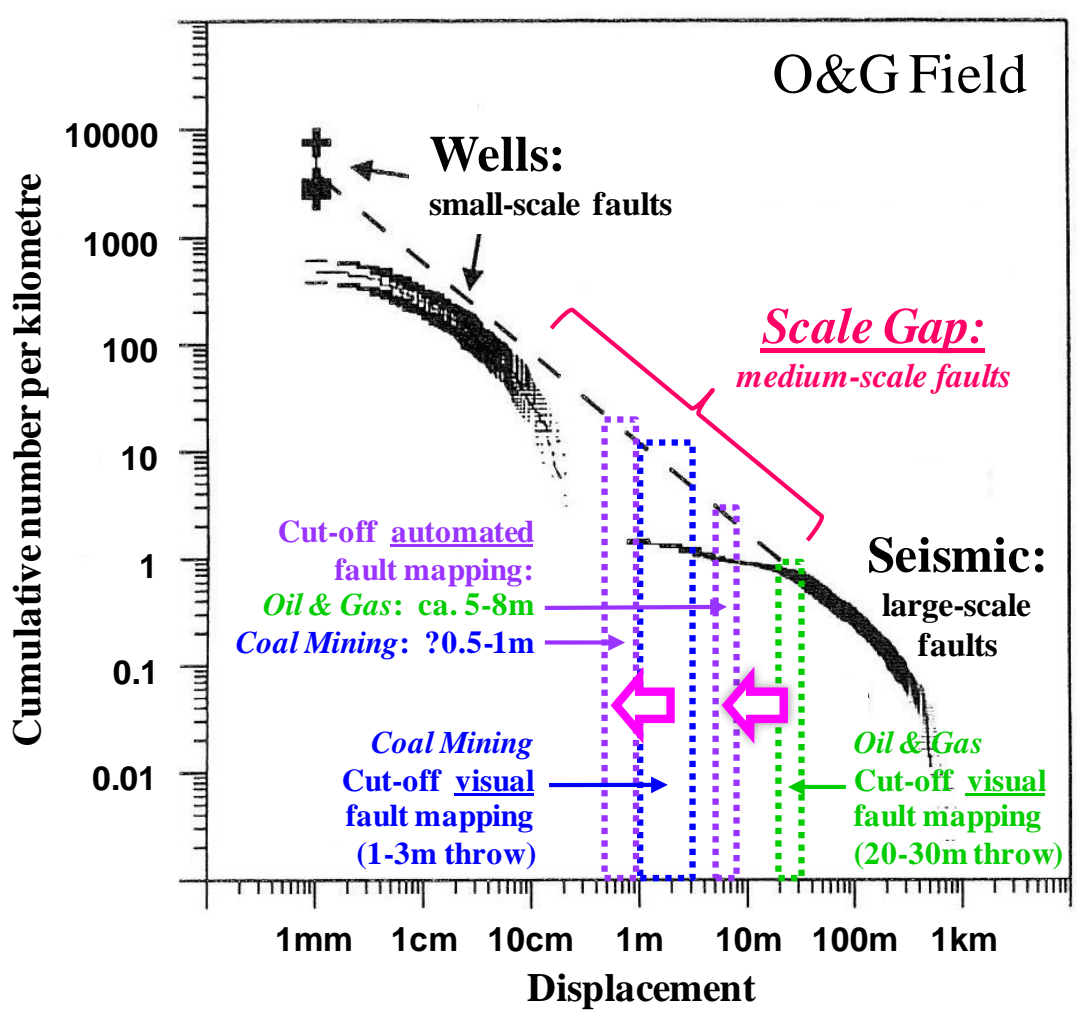

Figure 4 Comparison of visually mapped seismic fault throw data with well displacement data (modified from Needham et al., 1996). Displayed are also the cut-off ranges for visual fault mapping of faults from oil and gas and coal mining 3D surveys. These cut-offs can be lowered by automated fault extraction

- Calibration against faults and fractures identified in mines provides the best calibration opportunity as faults are not interpreted from other data, but are directly accessed and evaluated underground. There are also no scale gaps in mine fault data sets, which are affecting oil and gas seismic and well data (e.g. under-sampling of medium-sized faults in both oil and gas seismic and well data; Figure 4).

- Calibration against drilling observations, such as drill breaks, fluid losses or gains, gas kicks, HC shows, borehole instabilities, breakouts, well losses, microseismic events during hydraulic fracturing, etc.

- Calibration against mining observations, such as water inflow, outbursts, roof instability/collapse, rockbursts, fault reactivation during tunnelling, microseismic events during mining, etc.

- Calibration against well test observations, such as the presence of boundaries or baffles (or lack thereof), permeability pathways, etc.

- Calibration against hydrocarbon production observations, such as production logs, production enhancement through natural fracture system, water or gas channelling, evidence for presence of compartmentalisation, baffles or boundaries, reservoir pressure differentials between wells, 4D seismic data, etc.

- Calibration against mining production observations, such as fault corridors in ore deposits, spatial distribution of metal endowment, block/panel cave mining, caving induced subsidence, effectiveness of hydrofracs for fragmentation/caveability, coal seam offsets, pre-drainage of coal seam mine gas, etc. 
- Calibration through detailed discontinuity population analysis, incl. fault size and fault orientation analysis, fault throw and fault length frequency analysis (i.e. number of faults versus fault throw, or fault length), fault length versus throw analysis, histograms and rose diagrams, etc.

- Comparison of extraction results derived from different seismic vintages, e.g. time lapse seismic data, to test the repeatability of results. Stephenson et al. (2005) performed fault extractions (using Shell proprietary software) on two seismic surveys of different vintages that cover the same field. They found that all larger and most smaller fault bodies were co-located and showed the same structural trends in both seismic vintages. This result was seen as a strong confirmation that the extracted faults represented actual structural discontinuities rather than survey related artefacts.

Thorough and careful calibration helps to turn discontinuity data sets into calibrated fault and fracture network volumes, which can be utilised for further evaluation of identified issues in wells or mines, or for predicting and avoiding, or ensuring, fault and fracture intersections in future wells or mine shafts and tunnels.

\section{2 Benefits of automated fracture extraction}

The application of fracture extraction workflows in resource projects around the world has shown that properly calibrated fault and fracture network volumes can deliver a number of key benefits (Oppermann, 2010).

\subsubsection{Improved fault mapping objectivity, quality and reliability}

Manual fault mapping of 3D seismic data is often still performed in a 2D way, with fault picking being performed on vertical seismic sections and also horizontal time slices. In addition, fault picking is most times not performed on every single in-line or cross line, as this is very time-intensive. This interpretation mode is ultimately a relic of 2D interpretation times, and largely underutilises the information contained in 3D seismic data sets (Pepper and Bejarano, 2005). Fault detection with this approach can lead to incorrect geometries and even fault aliasing problems which normally only affect $2 \mathrm{D}$ seismic interpretation. The key problem associated with interpreter mapping, however, is that with decreasing fault throw (i.e. reflector offsets) visual interpretation becomes more and more challenging, subjective and uncertain, and visual fault mapping confidence decreases significantly. This is where automated fracture extraction allows a more objective and certain delineation of faults, particularly faults with small displacement, in true 3D space. This leads to an improved quality, achieves higher reliability compared to manual fault mapping and removes potential model-bias of an interpreter. Automated fracture extraction is based on the physical measurement of spatial variation in amplitude, phase and/or frequency content of 3D seismic data, and is as such free of bias and interpretation. While manual fault interpretation creates an interpretation of a measurement, fracture extraction creates a new measurement from an existing measurement. This measurement may prove to directly represent (deterministic) fracture networks, or can be used as a basis for further interpretation and model generation.

\section{2. 2 Faster evaluation}

Automated fracture extraction leads to a significant reduction in fault interpretation cycle time compared to manual (interpreter) mapping efforts. Hours or days of extraction work compare favourably to weeks or months of manual fault interpretation.

\section{2. 3 Improved structural definition}

Automated fracture extraction delivers an improved and more reliable structural definition of the subsurface and a better understanding of structural geometries and fault populations. Fracture properties such as distribution, orientation, size and fracture intensity can be quantified. Traditional mapping pitfalls, e.g. fault aliasing, oversimplification or the generation of geometrically unrealistic faults, can be avoided. 


\section{2. 4 New structural volumes}

A number of new structural fracture volumes can be generated for each extraction method: fracture network volumes, fracture network reflectivity volumes, fracture density volumes and fracture trend volumes (Figure 5). For all of these, sensitivity volumes can be also generated, reflecting different extraction parameterisations, for example to evaluate confidence in the picking of a discontinuity by a certain algorithm (Figure 6).
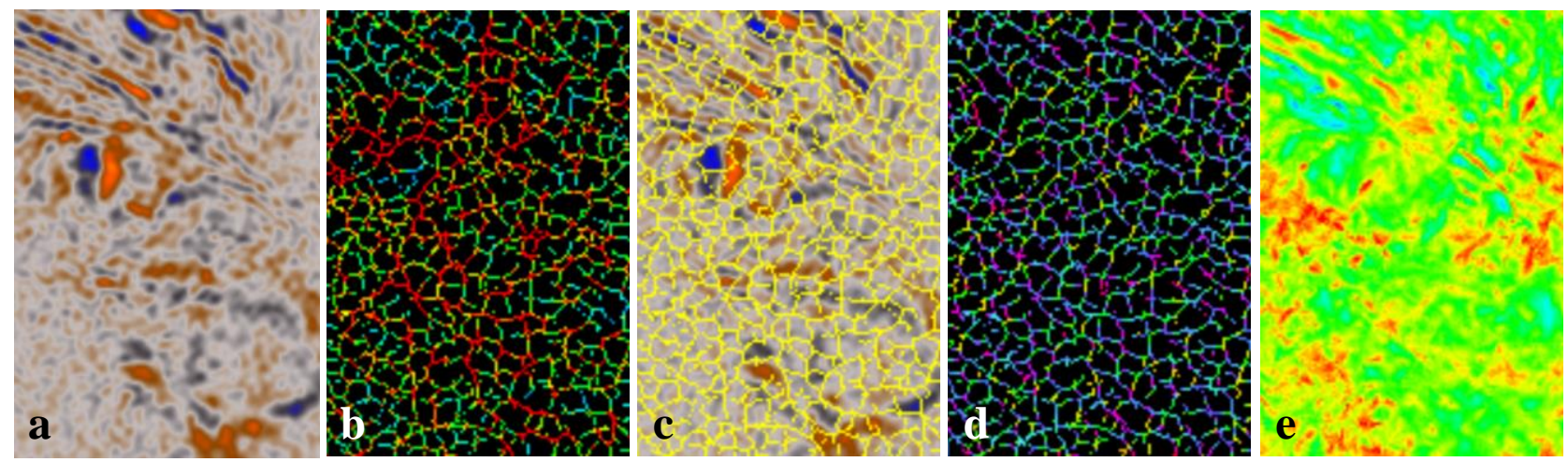

Figure 5 Comparison of reflectivity data with various high-resolution fault extraction results. Same depth slice through different volumes: (a) Reflectivity, (b) Fracture Network (red = high confidence,

blue = low confidence), (c) Fracture Network Reflectivity, (d) Fracture Trend (coloration according to strike orientation), (e) Fracture Density (red = high density, blue = low density) (Kevitsa Ni-Cu-PGE Mine, Finland)

F1
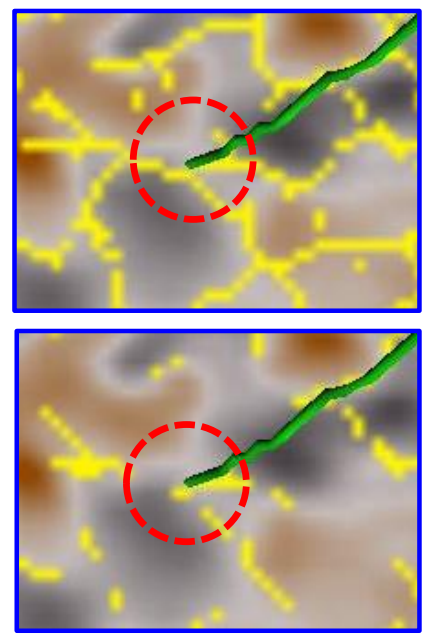

F2
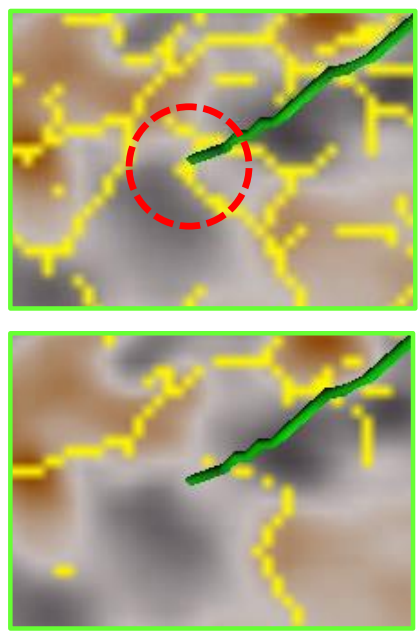

F3
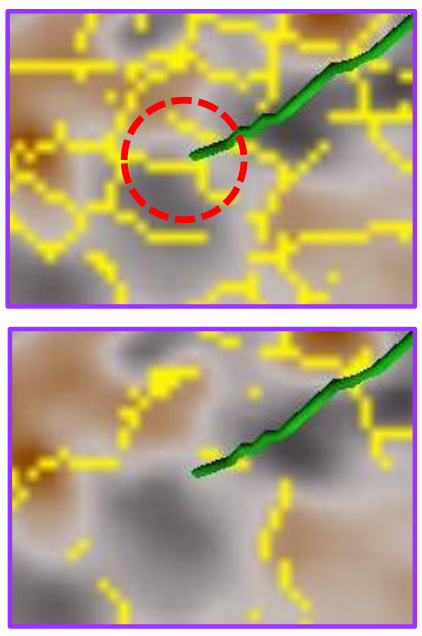

Figure 6 Three different high resolution fault extraction methods show a seismic fault that is directly associated with productivity in a basement well. Method F1 picks up this fault in both high resolution and high confidence extraction volumes. (Red circle highlights well intersection point with time slice)

\subsubsection{Quantified significance and confidence}

A further benefit of fracture extraction is that the uncertainty associated with manual fault interpretation can be removed and replaced with a measurable confidence value. Confidence values of seismic discontinuities express the confidence with which a certain algorithm has identified a seismic discontinuity in the data. This allows to evaluate and also quantify the significance of fractures and the confidence in fracture presence at any given location in the 3D seismic volume. As the confidence value is often a proxy for fault throw, it allows to quickly visualise and separate larger (higher confidence) from smaller (lower confidence) fractures. It is also possible to display 'equi-confident' fractures, i.e. all fractures that have been 
identified with the exact same confidence in a data volume. This is particularly useful in a production context, e.g. for visualising all fractures in the subsurface that have the exact same confidence as a fracture that was identified in a well and is linked with high metallogenic endowment.

\section{2. 6 Improved fault resolution}

The key benefit of automated fracture extraction is a marked increase in fault resolution, which results in a dramatic increase in the number of (medium-sized) faults that are identified from seismic (Figure 4). The resolution increase leads to much higher fault and fracture densities than were previously mappable or recognised, and it also identifies many fault penetrations in wells that were previously not recognised. As such, the technology helps to bridge the scale gap between seismic and well data. The very latest fracture imaging technology pushes fault resolution down to the true fault resolution of a particular data set, not the perceived fault resolution that is typically established by visual (Interpreter) mapping only. Most 3D surveys in the resource industries are therefore currently underutilised, as an entire medium-sized, 'subvisual' (but not sub-seismic) fault population could be extracted from already existing data with relatively little effort.

\subsection{Resource development optimisation}

The use of different algorithms during high-resolution fracture extraction allows to generate multiple (deterministic) 3D fault and fracture network models, which can subsequently be compared and calibrated with drilling, mining and production results (Figure 6). Calibration may result in the selection of a preferred (base case) extraction method. The significance of fractures and the confidence in fracture presence can be objectively and quantitatively evaluated by performing extractions with different parameterisations and by comparing the differences in results. With this new information, drilling, production, geotechnical and safety issues in existing wells or mines can be better understood, and future drilling, mining and production outcomes can be optimised. Fractures linked to drilling, mining and/or production risks or hazards can be avoided, and the planning and risk mitigation for underground mining operations can be significantly improved. Safer, cheaper and more successful wells can be drilled by designing future wells (especially deviated/horizontal wells) to stay clear of faulted or fractured zones previously not predictable on seismic, or by predicting zones in the well where fluid losses, potential kicks and borehole instabilities could occur. Future hydrocarbon wells can be optimally placed with respect to fluid boundaries or fluid conduits. Fault intersections can be planned to drain different fault compartments (in matrix-producing fields), or to access the productive natural fracture network ('sweet spots'). Fault-bounded mineralisations can be delineated from 3D seismic at high resolution and form the basis for detailed fractured rock mass characterisation and mineral resource evaluations. The prediction of primary fragmentation of rock mass in block and panel cave mines can be significantly improved. The data can be also used for the direct targeting of (hydrothermal) ore bodies with wells and mines.

\section{Conclusions}

The latest techniques and workflows in high-resolution Automated Fracture Extraction open up a new dimension in the detection, prediction and understanding of fault and fracture networks.

Results obtained from oil and gas as well as mining studies challenge widespread perceptions of what is seismically resolvable from 3D seismic data, and also offer groundbreaking new insights into the spatial distribution of fault and fracture networks and how they can affect the drilling and production of resources.

High-resolution fracture imaging provides exciting and significant opportunities to increase resource recoveries and reduce operational risks and costs, and is proposed as Best Practice tool for both mining and oil and gas resource development planning and execution. 


\section{Acknow ledgements}

The author would like to thank First Quantum Minerals Ltd for the permission to include visual material in this paper, and specifically Chris Wijns for his interest in the application of oil and gas technology to a mining asset. The author also thanks the following for their interest in the application of hi-res fracture extraction in mining and also helpful discussions about various mining issues: Winthrop Professor Yves Potvin (Australian Centre for Geomechanics, The University of Western Australia), Professor Gideon Chitombo (BRC, University of Queensland), Associate Professor Milovan Urosevic (WA School of Mines, Curtin University), Simon Brady and Andrew Willson (Anglo American Metall. Coal), Mike Webb (Anglo American Exploration), Clive Pickering (BHP Billiton Illawarra Coal), Oliver Kreuzer (Mitchell River Group) and Ronald Lachenicht (Newcrest Mining).

\section{References}

Admasu, F., Back, S. and Toennies, K. (2006) Autotracking of faults on 3D seismic data, Geophysics, Vol. 71, No. 6, pp. A49-A53.

Antonellini, M. and Aydin, A. (1994) Effect of faulting on fluid flow in porous sandstones: petrophysical properties, AAPG Bulletin American Association of Petroleum Geologists, Vol. 78, pp. 355-377.

Chopra, S. and Marfurt, K.J. (2007) Volumetric curvature attributes add value to 3D seismic data interpretation, The Leading Edge, July 2007, pp. 856-867.

Cocker, J., Urosevic, M. and Evans, B. (1997) A high resolution seismic survey to assist in mine planning, in Proceedings Exploration 97: Fourth Decennial International Conference on Mineral Exploration, A.G. Gubins (ed), pp. 473-476.

Gochioco, L.M. and Cotten, S.A. (1989) Locating faults in underground coal mines using high-resolution seismic reflection techniques, Geophysics, Vol. 54, No. 12, pp. 1521-1527.

Hoecker, C. and Fehmers, G. (2002) Fast structural interpretation with structure-oriented filtering, The Leading Edge, March 2002 , pp. 238-243.

Lonergan, L., Jolly, R.J.H., Rawnsley, K. and Sanderson, D.J. (2007) Fractured Reservoirs, Geological Society of London Special Publication 270, published 15 March 2007, L. Lonergan (author, editor), R.J.H. Jolly, K. Rawnsley, D.J. Sanderson (eds), 296 pages, ISBN-13: 978-1862392137.

Maerten, L., Gillespie, P. and Daniel, J-M. (2006) Three-dimensional geomechanical modeling for constraint of subseismic fault simulation, AAPG Bulletin, Vol. 90, No. 9, pp. 1337-1358.

Molinda, M. and Ingram, D.K. (1990) Effects of structural faults on ground control in selected coal mines in southwest Virginia, International Journal of Mining and Geological Engineering, Vol. 8, pp. 332-347.

Needham, T., Yielding, G. and Fox, R. (1996) Fault population description and prediction using examples from the offshore U.K., Journal of Structural Geology, Vol. 18, Nos 2/3, pp. 155-167.

Nelson, R.A. (2001) Geologic analysis of naturally fractured reservoirs, Elsevier Inc., 2nd edition.

Oppermann, R. (2010) A new method for high-resolution fault imaging delivers groundbreaking insights into drilling and production of resources, ASEG 21st International Geophysical Conference and Exhibition, Extended Abstract, Sydney.

Pepper, R. and Bejarano, G. (2005) Advances in seismic fault interpretation automation, Search and Discovery Article, \#40169, pp. 1-16.

Peters, T. and Hearn, S. (2001) The influence of coal-mine geology on seismic data quality in the Bowen Basin, in Extended Abstracts, ASEG 15th Geophysical Conference and Exhibition, August 2001, Brisbane.

Stephenson, B., Cassidy, P. and Warrlich, G. (2005) Novel use of seismic volume interpretation for fracture detection and modeling: examples from field in the Philippines and the Middle East, 14th SPE Middle East Oil and Gas Show and Conference, Bahrain, 12-15 March 2005, SPE 93759. 
Stress and structures 